\title{
Identification of proteins that interact with a plant nuclear protein using the yeast split-Trp sensor
}

\author{
Chikako Kubo, Yoshihiro Nomura, Naoki Matsuo, Hiroharu Banno* \\ Department of Environmental Biology, College of Bioscience and Biotechnology, Chubu University, \\ Kasugai, Aichi 487-8501, Japan \\ *E-mail: hirobanno@isc.chubu.ac.jp Tel: +81-568-51-6242 Fax: +81-568-52-6594
}

Received March 14, 2014; accepted April 24, 2014 (Edited by H. Shimada)

\begin{abstract}
The original yeast two-hybrid system is based on the functional reconstitution of the transcriptional activator of a reporter gene by means of the interaction between bait and prey proteins, which possess DNA-binding and transactivation domains, respectively. However, in certain cases, interaction between a fusion protein with a DNA-binding domain and a bait peptide can activate the transcription of a reporter gene, irrespective of the interaction between the bait and prey proteins. Therefore, this system cannot be used to screen for prey proteins that specifically interact with the "bait" because bait peptides initiate transactivation. We used a split-Trp sensor to identify protein interactions with the C-terminal region of Arabidopsis Enhancer of Shoot Regeneration 1(ESR1), which has transactivating capabilities in yeast cells. ESR1Interacting Candidate 1(EIC1) was identified using this system, and although its functions are unknown, EIC1 was observed to localize to nuclei when its GFP-fusion protein was expressed in onion epidermal cells. These results suggest that the splitTrp sensor could be useful in screening for proteins that interact with peptides that have transactivation domains in yeast cells.
\end{abstract}

Key words: ESR1, transcription factor, split-Trp sensor.

Protein-protein interactions play a central role in almost every biological process. The yeast two-hybrid system is very useful for identifying proteins that interact with a protein of interest. Various modified versions of the twohybrid system have been developed, including systems developed to study interactions between cytosolic or membrane proteins (Ferro and Trabalzini 2013). The original system is based on the transactivation of a reporter gene as a result of the interactions between bait and prey proteins with DNA binding and transactivation domains, respectively. However, when a bait protein initiates transactivation in yeast cells, its interaction with a fusion protein containing a DNA binding domain activates the transcription of a reporter gene, irrespective of its interactions with prey proteins. In these cases, this system cannot be used to screen for interacting proteins. Saccharomyces cerevisiae Trp1p is an $N$-(5'-phosphoribosyl) anthranilate isomerase, which is involved in the biosynthesis of tryptophan, and the split peptides of Trplp have been used to detect proteinprotein interactions by examining cell growth on media without tryptophan (Tafelmeyer et al. 2004). We used a split-Trp sensor to identify proteins interacting with transcription factors containing transcriptional activation domains in yeast nuclei. Very few applications of this system, including those in plant systems, have been reported (O'Hare et al. 2008).

We constructed pCtrp (DDBJ accession number $\mathrm{AB} 921961$ ) and pNtrp (DDBJ accession number AB921962) vectors (Figure 1) for this study. The myristylation signal sequence in pMyr (Agilent Technology) was replaced by the C-terminal region of TRP1 (Ctrp; amino acid residues 45-224) with Myc-tag in the N-terminus to generate pCtrp. NLSVP16 in pVP16 (Hollenberg et al. 1995) was replaced by the N-terminal region of TRP1 (Ntrp; amino acid residues 1-44) with HA-tag to generate pNtrp. Ctrp was expressed under the control of a galactose-inducible promoter(GAL1 promoter), and Ntrp was expressed under the control of a constitutive promoter from $A D H 1$. In this system, the enzymatic activities of the protein can be restored by reconstitution of Trplp when the bait protein interacts with the prey protein. Prior to screening, we investigated whether a combination of known interacting proteins could synthesize tryptophan using the split-Trp sensor. Arabidopsis IAA12 and TOPLESS(TPL) proteins have been reported to physically interact (Szemenyei et al. 2008), and the auxin- 


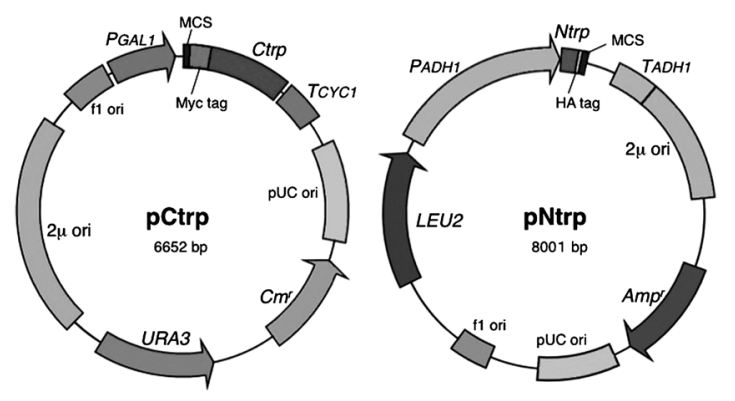

Figure 1. Plasmid maps of pCtrp and pNtrp. pCtrp is a split-Trp bait vector harboring the chloramphenicol resistance gene $(\mathrm{Cm})$ and URA3, which are bacterial and yeast selectable markers, respectively, as well as the high copy number $2 \mu$ yeast replication origin $(2 \mu$ ori $)$. The diagram shows the location of the yeast GAL1 promoter $\left(P_{G A L 1}\right)$ controlling the expression of Ctrp in addition to a multiple cloning site (MCS) for the insertion of bait cDNA, Myc-tag sequence for protein detection and yeast CYCLIN 1 terminator $\left(T_{C Y C I}\right)$, respectively. pNtrp is a prey vector harboring the ampicillin resistance gene (Amp) and LEU2 for bacterial and yeast selectable markers, respectively, as well as the $2 \mu$ ori. The expression of Ntrp is controlled by the yeast alcohol dehydrogenase 1 $(A D H 1)$ promoter $\left(P_{A D H 1}\right)$ followed by an HA-tag for protein detection, an MCS for insertion of prey cDNAs and the $A D H 1$ terminator $\left(T_{A D H 1}\right)$.

responsive proteins IAA12 and TPL are involved in the negative regulation of transcription, thereby localizing to the nucleus. In the present study, TPL and IAA12 cDNA were inserted into pCtrp and pNtrp, respectively (pCtrp::IAA12 and pNtrp::TPL). Yeast EGY45 (MAT $\alpha$ ura3 his3 trp1 leu2 6LexAop-LEU2) cells harbored combinations of empty vectors and either pCtrp::IAA12 or pNtrp::TPL (Figure 2). In contrast, cells harboring pCtrp::IAA12 and pNtrp::TPL grew on galactose medium but not glucose medium, indicating that the split-Trp was functional in a galactose-dependent manner. These results suggest that split-Trp can function as a sensor of protein-protein interactions in the nucleus.

ESR1, an Arabidopsis transcription factor involved in shoot regeneration in tissue culture (Matsuo et al. 2011), has a transactivation domain that is functional in yeast cells (our unpublished results). The ESR motif in the C-terminal region of ESR1 has transactivation ability in plant (Nomura et al. 2009) and yeast cells (our unpublished results). This motif is essential for ESR1 activities, and ESR1 loses activity when it is replaced with a strong transactivation peptide, such as VP16, suggesting that the ESR motif has additional functions other than transactivation. We hypothesized that the C-terminal region of ESR1 interacts with other nuclear proteins involved in regulating shoot regeneration. To identify proteins interacting with the ESR motif, the nuclear split-Trp system was applied. For this purpose, the C-terminal region of ESR1 (199-328), including the ESR motif was fused to the MPPKKKRKV sequence, the nuclear localization signal(NLS) of the large SV40 $\mathrm{T}$-antigen with an initiation codon. The hexamer protein SV40 is known to function in plant cells (Raikhel 1992) and its gene was inserted into pCtrp (pCtrp::NLS-

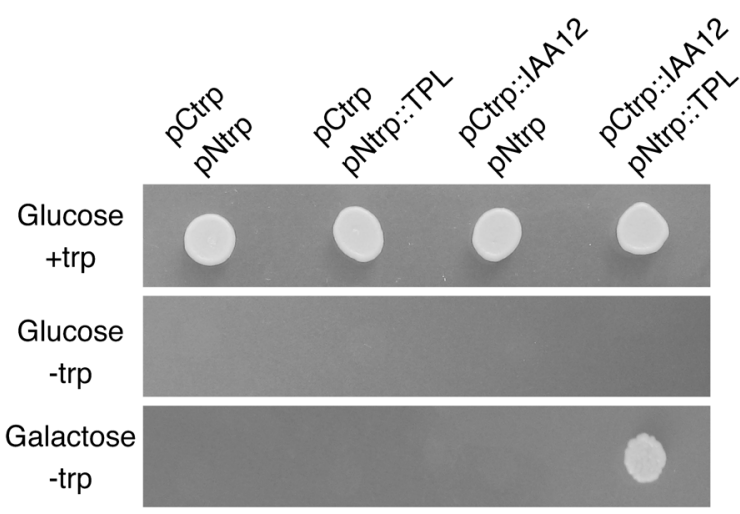

Figure 2. Growth assays of yeast strains expressing Ctrp-IAA12 and Ntrp-TPL. In these experiments, IAA12 was fused to the Ctrp protein fragment and TPL was fused to the Ntrp protein fragment. A colony of yeast EGY48 cells harboring the indicated plasmids was suspended in water $(1 \mathrm{ml})$ and $5 \mu \mathrm{l}$ was spotted on medium with or without $20 \mathrm{mg} / \mathrm{l}$ L-tryptophan (trp) and either $2 \%$ glucose or $2 \%$ galactose at $23^{\circ} \mathrm{C}$. The images were taken after 8 day.

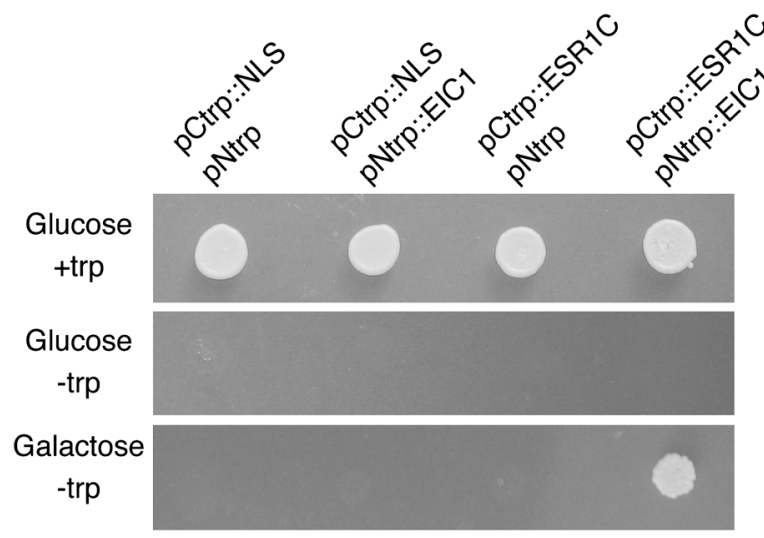

Figure 3. Growth assays of yeast strains expressing Ctrp-ESR1C and Ntrp-EIC1. In these experiments, ESR1C was fused to the Ctrp protein fragment and EIC1 was fused to the Ntrp protein fragment. An NLS from SV40 was fused to the Ctrp (pCtrp::NLS) as a negative control. A colony of yeast EGY48 cells harboring the indicated plasmids was suspended in water $(1 \mathrm{ml})$ and $5 \mu \mathrm{l}$ was spotted on medium with or without $20 \mathrm{mg} / \mathrm{l} \mathrm{L}$-tryptophan (trp) and either $2 \%$ glucose or $2 \%$ galactose at $23^{\circ} \mathrm{C}$. The images were taken after 8 day.

ESR1C) because the NLS of ESR1 was within the $\mathrm{N}$-terminal region. The cDNA was synthesized from mRNA obtained from cultured Arabidopsis tissues (Banno et al. 2001) and subsequently inserted into the pNtrp vector to construct a cDNA library. Yeast cells (EGY45) harboring pCtrp::NLS-ESR1C and a cDNA library in pNtrp were cultured on a galactose medium at $23^{\circ} \mathrm{C}$ for 10 day because the split-Trp sensor functions only at low temperatures and the cells proliferate very slowly. Twenty-five colonies were chosen and their growth was examined on a glucose medium. Eight clones did not grow on the glucose medium. Some of the 17 clones that showed galactose-independent growth may contain cDNA homologous to Arabidopsis TRP1. However, this requires further investigation. Another possible reason for the galactose-independent growth 


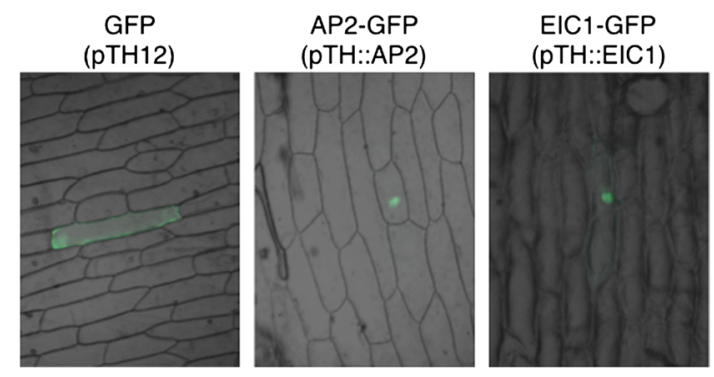

Figure 4. Subcellular localization of GFP-fusion protein with EIC1. The AP2/ERF domain of ESR1 containing an NLS or full-length EIC1 was fused to GFP in a GFP expression vector, and pTH12 was under the control of the cauliflower mosaic virus $35 \mathrm{~S}$ promoter. AP2 (the AP2/ ERF domain of ESR1) or full-length EIC1 was fused to the N-terminus of GFP (pTH::AP2 or pTH::EIC1). These constructs were bombarded into onion epidermal cells and images were taken after $24 \mathrm{~h}$. Bright field images and GFP fluorescent images were merged as shown.

could be the reversion of the $\operatorname{trp} 1$ mutation because $\operatorname{trp} 1$ in yeast EGY48 cells has a point mutation. pNtrp vectors with cDNA were recovered from cells, demonstrating galactose-dependent growth, and the cDNA sequences were analyzed. Based on their cDNA sequences, the clones were identified to be one of the following five genes: At2G05440 (3 clones); At3G02220 (2 clones); At3G51780 (2 clones); and At4G28703 (1 clone). All of these genes encode proteins with unknown functions, although At2G05440, At3G51780, and At3G51780 encode glycine-rich protein 9, AtBAG4, and RmlC-like cupins superfamily protein, respectively. Among these candidate proteins, we investigated At3G02220 (ESR1Interacting Candidate 1; EIC1), which has a predicted amino acid sequence containing basic stretches that may function as a NLS (Lange et al. 2007). Figure 3 shows the interaction between NLS-ESR1C and EIC1. To exclude the possibility that EIC1 interacted with NLS, pCtrp::NLS was used as a negative control. Cells harboring combinations of either pCtrp::NLS or pCtrp::NLS-ESR1C and pNtrp or pNtrp-EIC1, respectively, were grown on the indicated medium. Cells harboring pCtrp::NLSESR1C and pNtrp::EIC1 grew in a galactose-dependent manner, indicating EIC1 interacted with the C-terminal region of ESR1.

To examine its cellular localization, full-length EIC1 cDNA was amplified by PCR from a cDNA library and fused to the N-terminus of GFP. GFP (pTH12), AP2-GFP (pTH::AP2) , or EIC1-GFP (pTH::EIC1) was transiently expressed in onion epidermal cells after introducing the constructs by particle bombardment. The AP2/ERF domain of ESR1 (AP2) containing an initiation codon was used as a positive control because it is known to localize to nuclei (Matsuo and Banno 2008). Onion cells were observed under a fluorescence microscope $24 \mathrm{~h}$ after bombardment. As shown in Figure 4, only GFP was distributed throughout the cells, whereas EIC1-GFP as well as AP2-GFP specifically targeted the nuclei. These results support the hypothesis that EIC1 may interact with ESR1 in the nucleus of a plant cell.

In conclusion, we identified EIC1 as a candidate that can interact with the C-terminal region of ESR1 using the split-Trp sensor. EIC1 localized specifically to nuclei of onion cells when transiently expressed; however, whether these interactions are directly or indirectly mediated by yeast factors remains to be elucidated using purified recombinant proteins. Our results suggest that the splitTrp sensor can be used to identify nuclear proteins with transactivation domains.

\section{Acknowledgements}

This research was supported by a Grant-in-Aid for General Scientific Research (no. 25440143 to H. B.) from JSPS (Japan Society for the Promotion of Science).

\section{References}

Banno H, Ikeda Y, Niu QW, Chua NH (2001) Overexpression of Arabidopsis ESR1 induces initiation of shoot regeneration. Plant Cell 13: 2609-2618

Ferro E, Trabalzini L (2013) The yeast two-hybrid and related methods as powerful tools to study plant cell signalling. Plant Mol Biol 83: 287-301

Hollenberg SM, Sternglanz R, Cheng PF, Weintraub H (1995) Identification of a new family of tissue-specific basic helixloop-helix proteins with a two-hybrid system. Mol Cell Biol 15: 3813-3822

Lange A, Mills RE, Lange CJ, Stewart M, Devine SE, Corbett AH (2007) Classical nuclear localization signals: definition, function, and interaction with importin alpha. J Biol Chem 282: 5101-5105

Matsuo N, Banno H (2008) The Arabidopsis transcription factor ESR1 induces in vitro shoot regeneration through transcriptional activation. Plant Physiol Biochem 46: 1045-1050

Matsuo N, Makino M, Banno H (2011) Arabidopsis ENHANCER OF SHOOT REGENERATION (ESR) 1 and ESR2 regulate in vitro shoot regeneration and their expressions are differentially regulated. Plant Sci 181:39-46

Nomura Y, Matsuo N, Banno H (2009) A domain containing the ESR motif in ENHANCER OF SHOOT REGENERATION 1 functions as a transactivation domain. Plant Biotechnol 26: 395-401

O'Hare H, Juillerat A, Dianiskova P, Johnsson K (2008) A splitprotein sensor for studying protein-protein interaction in mycobacteria. J Microbiol Methods 73: 79-84

Raikhel N (1992) Nuclear targeting in plants. Plant Physiol 100: $1627-1632$

Szemenyei H, Hannon M, Long JA (2008) TOPLESS mediates auxin-dependent transcriptional repression during Arabidopsis embryogenesis. Science 319: 1384-1386

Tafelmeyer P, Johnsson N, Johnsson K (2004) Transforming a (beta/alpha)8-barrel enzyme into a split-protein sensor through directed evolution. Chem Biol 11: 681-689 